\title{
CUANDO EL PEREGRINO COMULGA CON EL TURISMO: SOBRE LA INCLUSIÓN DE UNA DIMENSIÓN TURISMOLÓGICA EN LAANTROPOLOGÍA DEL PEREGRINAR
}

\author{
Martín FABREAU*
}

Resumo: Este texto procura mostrar cómo el abordaje antropológico de las peregrinaciones pasó a incorporar una dimensión turismológica en sus modelos de análisis, trascendiendo así la dicotomía 'sagrado/profano' en la que turismo y peregrinación aparecen como prácticas opuestas e incompatibles. Dicho pasaje quedará de manifiesto a través de la presentación de los principales modelos con que la Antropología ha abordado la institución del peregrinaje; una práctica espacial que debido a sus características tan particulares presenta varios desafíos tanto teóricos, como metodológicos. A partir del modelo clásico y hegemónico de Victor y Edith Turner, que duró más una década y en el que se concibe al peregrinar como un fenómeno liminoide y propiciador antiestructura o communitas, se pasará hacia modelos contemporáneos que van más allá del ámbito de lo sagrado y hacen foco en las interfaces entre esta práctica y otras dinámicas de movilidad como lo es el turismo y todo lo que esta actividad implica. En lugar de concebir las figuras del peregrino y del turista como diferentes y antagónicas, representando una lo sagrado y otra lo profano, comienza a tomarse cada vez más en serio la idea de que un mismo sujeto puede ser peregrino y turista, o que el peregrinar en la actualidad además de comportar en gran medida una dimensión sagrada inevitablemente también lleva consigo una dimensión que remite a diversos fenómenos culturales vinculados al consumo y también al tiempo libre. Finalmente, se presentarán algunos correlatos empíricos cuyos abordajes son emblemáticos de este cambio de visión, buscando mostrar algunos aportes de la Antropología del Peregrinar para pensar las peregrinaciones desde los estudios turísticos.

Palavras chave: Turismo; Antropología; Movilidad; Sagrado; Peregrinación.

\section{WHEN THE PILGRIM COMMUNES WITH TOURISM: ABOUT THE INCLUSION OF A TOURISMOLOGIST DIMENSION ON THE ANTHROPOLOGY OF PILGRIMAGE}

Abstract: This paper aims to showing how, the anthropologic approach of pilgrimage went on to incorporate a touristic dimension in its framework, thus transcending the 'sacred/profane' dichotomy under which tourism and pilgrimage appear as practices which are both opposed and incompatible. This will be evident through the presentation of the main models with which Anthropology has approached the institution of pilgrimage; a spatial practice that, due to its particular characteristics, presents several theoretical and methodological challenges. Based on the classic and hegemonic model, by Victor and Edith Turner, for more than a decade, in which the pilgrimage is understood as a preliminary and propitious phenomenon of antistructure or communitas, we will move on to contemporary models that go beyond the scope of the sacred and focus on the interfaces between this practice and other mobility dynamics such as tourism itself and everything that this activity implies. Instead of conceiving the figures of both the pilgrim and that of the tourist as two different and antagonistic figures, the former representing the sacred and the latter the profane, the idea of a subject being both a pilgrim and a tourist, begins to gain ground. Alongside this, the current conceptions of pilgrimage transcend the sacred spheres, entailing various cultural phenomena related to activities such as that of consumption and of spare time. Finally, it will be presented a few empirical correlates whose approaches are representative of this shift in the view, seeking to show some of the contributions made by the Anthropology of Pilgrimage, in order to conceive pilgrimage from the touristic point of view and approach.

Key words: Tourism; Anthropology; Mobility; Sacredness; Pilgrimage.

\section{QUANDO O PEREGRINO COMUNGA COM O TURISMO: SOBRE A INCORPORAÇÃO DE UMA DIMENSÃO TURISMOLÓGICA NA ANTROPOLOGIA NO PEREGRINAR}

Resumo: Este texto procura mostrar como a abordagem antropológica das peregrinações passou a incorporar uma dimensão turismológica em seus modelos de análise, transcendendo a dicotomia 'sagrado/profano' onde turismo e peregrinação aparecem como práticas opostas e incompatíveis. Isso ficará evidente através da apresentação dos principais modelos com que a Antropologia tem abordado a instituição da peregrinação; uma prática espacial que devido às suas características tão particulares apresenta variados desafios tanto teóricos quanto metodológicos. A partir do modelo clássico e hegemônico, de Victor e Edith Turner, durante mais de uma década, no qual se entende o peregrinar como um fenômeno liminar y propiciador de antiestrutura ou communitas, se passará a modelos contemporâneos que vão além do âmbito do sagrado e fazem foco nas interfaces entre esta prática e outras dinâmicas de mobilidade como é o próprio turismo e tudo o que esta atividade implica. Ao contrário de entender as figuras do peregrino e do turista como diferentes e antagônicas, representando uma o sagrado e outra o profano, começa a ser levada cada vez mais em conta a ideia de que um mesmo sujeito pode ser peregrino e turista, ou que o peregrinar na atualidade além de comportar em grande medida uma dimensão sagrada inevitavelmente também leva consigo uma dimensão que remete a diversos fenômenos culturais vinculados ao consumo e ao lazer. Finalmente, apresenta-se alguns correlatos empíricos onde as suas abordagens resultaram emblemáticas a esta mudança de visão, procurando mostrar alguns aportes da Antropologia do Peregrinar para pensar as peregrinações desde os estudos turísticos.

Palavras-chave:Turismo; Antropologia; Movilidade; Sagrado; Peregrinação.

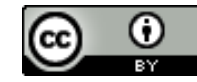

Licenciada por Creative Commons 4.0/Internacional CCBY 4.0
* Doctor y Magíster, ambos por el Programa de Pós-Graduação em Antropologia de la Universidade Federal de Pernambuco (PPGA-UFPE). Licenciado en Ciencias Antropológicas por la Facultad de Humanidades y Ciencias de la Educación- Universidad de la República, Uruguay (FHCE - UDELAR). Profesor Adjunto del Área de Estudios Turísticos, Facultad de Humanidadesy Ciencias de la Educación-Universidad de la República, Uruguay (AET - FHCE - UDELAR). Miembro de la Asociación Uruguaya de Antropología Social y Cultural (AUAS). Lattes: http://lattes.cnpq.br/6313601380577798. Orcid: https://orcid.org/0000-0003-4642-9817?lang=es .E-mail: fabreau@gmail.com 


\section{INTRODUCCIÓN: MOVILIDAD COMO 'SEÑAL DE LOS TIEMPOS'}

Viajeros, migrantes, turistas, peregrinos, conferencistas, estudiantes, convertidos, reconvertidos. $\mathrm{Si}$ hay un signo que parecería caracterizar la contemporaneidad es el de la movilidad. Podría señalarse no obstante que ninguna de estas figuras es reciente; de ahí cabría entonces la inversión de los términos y señalar que lo novedoso en estos tiempos no es tanto la movilidad como el utilizar la movilidad para mirar estos tiempos.

\begin{abstract}
Mobilities are central to the structuring of people's lives. In many parts of the world, mobility is an important way of belonging to today's society. We can identify multiple types of "movers": tourists and pilgrims; migrants and refugees; diplomats, businesspeople, and those working for international organizations; missionaries, NGO workers, and people belonging to the most diverse transnational networks; students, teachers, and researchers; athletes and artists; soldiers and journalists; children and partners (and service personnel) accompanying the aforementioned people; and those in the traffic and transport industries who move people (including themselves) across the globe (Salazar, 2018: 154).
\end{abstract}

En la tradición antropológica hay profusas evidencias de este cambio de foco; Clifford (1999a), por ejemplo, establece que la conexión intercultural es la norma y lo ha sido desde hace mucho tiempo, o Hannerz (1997) establece que la noción de flujo cultural hace un buen tiempo ya que es utilizada.

\footnotetext{
The world as known today exists as testimony to, and evidence of, the fact that people travel. [...] The factors that shaped early patterns of travel were fundamentally directed by basic human needs (finding food and shelter), exchange (trade), relationships with natural phenomena (developing new settlements, escaping droughts or floods, etc.), and the result of conquest and conflict (occupation, expulsion, forced migration, and resettlement). Such factors still exert considerable influence on a large proportion of the world's population today, with contemporary pilgrimage routes relatively easy to identify, and tourism frequently building on established trading relationships and patterns of diaspora and relocation (Jamal \& Robinson, 2009: 3).
}

Mirar la realidad social a través de la lente de la movilidad, significa entonces bastante más que incorporar apenas el desplazamiento a la hora de analizar situaciones o estudiar situaciones en las que prima el movimiento espacial; implica ante todo la concepción de un nivel teorético en el que el movimiento además de ser foco es modelo; además de ser objeto es metáfora que trasciende lo espacial para pasar a connotar también el fluir de la cultura.

The ascendance of theoretical frameworks in anthropology that emphasize mobility and border crossings over previous approaches that analyzed the world as though it were divided into static and bounded social units has had major implications for research on tourism, pilgrimage, and other forms of travel. A cluster of related themes-including travel and travelers, displacement, new cosmopolitans, postmodern "nomads," borders, transculturalism, and migrations-have come to dominate much of the literature in anthropology and related fields since the 1990s. These new metaphors reflect a world in which there is an unprecedented number of people traveling across wide distances as immigrants, refugees, labor migrants, or temporary sojourners as well as tourists and pilgrims (Bandone \& Roseman, 2004: 8-9)

De esta manera, Clifford utilizará el concepto de "viaje" como término de "comparación cultural" y por ende y pasa a se considerarlo como "una amplia gama de prácticas materiales y espaciales que producen conocimientos, historias, tradiciones, [...] y otras expresiones culturales" (Clifford, 1999b: 51). O Bauman desde otra tradición utilizará la metáfora del peregrinaje para significar este momento histórico portador de cambios permanentes (Coleman \& Eade, 2004), más allá de sus clásicas figuras de turistas y vagabundos, y de sus tantas metáforas relativas a lo líquido (Bauman, 1999; Ribeiro, 2017), imágenes que aluden a otros aspectos de la movilidad. También Hervieu-Léger desde la Sociología de la Religión utilizará la figura del "peregrino" para integrar la movilidad tanto interior como exterior en las nuevas configuraciones de la creencia en la modernidad religiosa (Hervieu-Léger, 2005). O yendo más allá, Lash y Urry (1998) profundizan en el modelo de una sociedad posfordista y posmoderna signada por el establecimiento de flujos de sujetos y objetos; "el 
orden global contemporáneo, o el desorden, es así una estructura de flujos, un conjunto des-centrado de economías de signos en un espacio" (Lash \& Urry, 1998: 17).

Es pues este contexto teórico y metateórico de la movilidad el que servirá de marco para este texto y que girará en torno de la práctica espacial del peregrinaje y su relación con prácticas que van más allá del ámbito de lo sagrado y que apuntan a la existencia de una dimensión turística en dicha práctica.

El lugar de enunciación será, no el de la turismología, sino el de la antropología enfocada en fenómenos religiosos, la cual, tratando de encontrar un abordaje más cabal que dé cuenta que el peregrinar comienza a habilitar nuevas interfaces con los estudios turísticos. En lugar de concebir las figuras del peregrino y del turista como diferentes y antagónicas, representando una lo sagrado y otra lo profano, comienza a tomarse cada vez más en serio la idea de que un mismo sujeto puede ser peregrino y turista, o que el peregrinar en la actualidad además de comportar en gran medida una dimensión sagrada también lleva inevitablemente consigo una dimensión que remite a diversos fenómenos vinculados al consumo, al viaje y también al tiempo libre. El foco de este trabajo estará puesto en ese cambio en la mirada de los estudios antropológicos y en la adopción de modelos que en líneas generales rigen hasta el presente.

Trabajar con la práctica del peregrinaje ha sido y es tan complejo como trabajar con la noción de peregrinaje. ¿Qué se entiende por tal cosa? ¿Cómo englobar bajo un mismo concepto prácticas que revisten formas tan diferentes en diversas tradiciones religiosas y en diversos momentos históricos? ¿Hasta qué punto corresponde la construcción de una categoría etic que englobe todas las modalidades de peregrinación?

Como punto de partida bajo la forma general de peregrinaje podría reconocerse aproximadamente una práctica que comporta un punto de partida profano, un viaje particularmente cargado de significación religiosa y un lugar sagrado a modo de punto de llegada:

Peregrino é 'aquele que viaja para um lugar sagrado num ato de devoção religiosa', enquanto 'peregrinação' é 'a viagem de um peregrino'. 'Sagrado', (...) significa 'consagrado ou estimado por uma divindade (...) dedicado, reservado ou apropriado a alguma pessoa ou propósito; consagrado por associação religiosa, santificado (Turner, 2008a: 161).

Si bien esta práctica tiene casi la edad de las religiones y hoy día mueve millones de personas en el mundo, la antropología ha demorado sensiblemente en asumir el desafío de dar cuenta de ese objeto (Coleman, 2002). Las razones tienen que ver con la insuficiencia de las herramientas tanto metodológicas como teóricas y hasta metateóricas de la antropología/etnografía tradicional. El principal problema remite a la imposibilidad de esas herramientas para trabajar el movimiento (Coleman, 2002). De allí surgen algunos desafíos metodológicos sobre cómo realizar un trabajo de campo tradicional, encuadrado, delimitado en el contexto de un viaje sagrado. Así como también desafíos metaetnográficos como por ejemplo, de qué manera poder representar mediante 'antropologías de aldea' prácticas individuales o grupales de personas en tránsito provenientes de varios lugares estableciendo las más de las veces vínculos evanescentes (Hervieu-Léger, 2005). A lo anterior se le suma problemas adicionales que tienen que ver con la comparación; problemas ya conocidos que se presentan también cuando se trabaja sobre la práctica ritual.

A los efectos de esta presentación mostraré en primer lugar cómo ha sido tradicionalmente abordada la práctica del peregrinaje en antropología, para luego mostrar cómo han ido cambiando tanto la forma de modelarla como el cúmulo de relaciones donde se hace foco. Para dicho itinerario me basaré en el desarrollo realizado por Coleman (2002) y por Coleman y Eade (2004) por considerarlos los más influyentes en la actualidad aunque, sin por ello desconocer el aporte de Badone y Roseman (2004) quienes en la misma época y en la misma dirección teórica que autores como Coleman, además de Eade y Sallnow, discutiendo los abordajes tradicionales e incluyendo aspectos como el consumo, el turismo, la globalización y la dimensión performática de tales dislocamientos, abordaron el tema reuniendo una importante variedad de estudios de caso. Por último, mostraré algunos ejemplos de dicha transformación, enfocados principalmente en el caso brasileño. 
Tomados en conjunto, estos modelos de comienzos de la década de 2000, así como algunos estudios de caso que serán presentados en este texto y que devinieron emblemáticos, orientan hasta el día de hoy la mirada a la hora de trabajar con este tipo de fenómenos.

A través de esta posible trayectoria conceptual y sus correlatos empíricos, procuro dejar en evidencia dos aspectos: en primer lugar, la apertura de los modelos antropológicos clásicos hacia otras tradiciones teóricas y disciplinares para una mejor comprensión de las peregrinaciones y por otro algunos aportes de la antropología del peregrinar para pensar este fenómeno desde los estudios turísticos.

\section{MARCO TEÓRICO}

\subsection{Peregrinaje y Communitas: el Modelo Turneriano}

En un contexto en el que la antropología de la religión no reparaba en la institución del peregrinaje, la notable excepción fue el importante trabajo de Victor y Edith Turner, Image and Pilgrimage in Christian Culture (1978), en el cual se concebía la instancia del peregrinar como un fenómeno liminoide, y en tanto tal, propiciador 'antiestructura' o 'communitas'. En palabras de los autores,

the present book is an attempt to examine in some detail what we consider to be one characteristic type of liminality in cultures ideologically dominated by the 'historical', or 'salvation', religions (Turner \& Turner, 1978: 3).

En este texto, considerado un clásico y una referencia obligatoria, los autores abordan la categoría analítica "peregrinación" como una institución, y muestran que esta práctica es tan antigua como las principales religiones al tiempo que es parte fundamental. El modelo propuesto a través de los análisis de las peregrinaciones contemporáneas les otorgó a estos eventos un nuevo alcance, profundizando en la comprensión de la vida social en general (Carneiro, 2004).
En un texto de 1971 y que marca un antecedente de ese libro, Victor Turner señala que su cometido consiste en realizar un estudio comparativo de los procesos de peregrinación en las religiones históricas más emblemáticas tanto sincrónica como diacrónicamente (Turner, 2008a), también señala que se encuentra ampliando su registro de tal modo de poder abordar además religiones antiguas y manteniendo la esperanza de dar cuenta de eventuales prácticas de peregrinaje en las sociedades etnográficas. Para el autor las peregrinaciones pueden ser consideradas como

uma rede interligada de processos, cada qual envolvendo uma viagem de e para um local específico. Estes locais eram [são] lugares onde, de acordo com os fiéis, ocorrerá alguma manifestação de poder divino ou sobrenatural, aquilo que Mircea Eliade chamaria de 'teofania' (Turner, 2008a: 176).

Ya aquí Turner entiende a las peregrinaciones como fenómenos liminares, es decir como una instancia que propicia lazos de communitas, o sea antiestructurales; "indiferenciados, igualitários, diretos, não-racionais, existenciais" (Turner, 2008b: 255), caracterizados por un contexto de espontaneidad, inmediatez, poco o nada normativizado, evanescente 2 .

De lo anterior, las peregrinaciones son entendidas como fenómenos liminoides que generan encuentros sin constricciones jerárquicas. En relación a lo anterior, Coleman (2002: 356) agrega: "Communitas described the individual pilgrim's temporary transition away from mundane structures and social interdependence into a looser commonality of feeling with fellow visitors."

Cabe señalar que el concepto de liminaridad utilizado por Turner proviene de Arnold Van Gennep, quien la entendía como una fase intermedia dentro de un rito de pasaje en el que las categorías ordinarias de la vida social cotidiana quedan en suspenso;

\footnotetext{
1Existen algunas formas recurrentes de caracterizar la oposición entre 'estructura' y 'communitas'; de hecho El Proceso Ritual (1969) gira en torno a este cometido. Aquí no obstante se trabajará con un texto posterior y sintético de 1974 titulado Metáforas de la antiestructura en cultura religiosa. 2El pensamiento de Turner es indudablemente original aunque es claro que también en él existen marcas de sus predecesores. En este punto concreto y pese a que el autor nunca sea explícito, es posible hacer algún tipo de conexión entre el contexto de communitas y las situaciones excepcionales y pasajeras de efervescencia de las que hablaba Durkheim en Las formas elementales de la vida religiosa: "(...) as interações sociais tornam-se muito mais frequentes e mais ativas. Os indivíduos procuram-se e reúnem-se mais. Resulta daí uma efervescência geral, característica das épocas revolucionárias ou criadoras. Ora, essa atividade maior tem como efeito estimulação geral das forças individuais. Vive-se mais e de maneira diferente do que normalmente. As mudanças não são apenas de nuanças e de graus; o ontem torna-se outro" (Durkheim, 2008: 265). Y cabe señalar también que ya este autor pensaba estos estados oponiéndose a fuerzas normalizantes: "Além dessas forças em estado livre que vêm continuamente renovar as nossas, existem aquelas que estão fixadas nas técnicas e tradiç̃os de toda espécie que utilizamos" (Durkheim, 2008: 266).
} 
ele insistia que em todos os movimentos ritualísticos havia ao menos um momento em que aqueles seres que agiam de acordo com um script cultual liberavam-se das exigências normativas, momento este o qual eles estavam, de fato, betwix and between, (...) Neste ínterim da 'liminaridade' existe a possibilidade de se ficar fora não somente da sua própria posição social, mas de todas as posições sociais, e de se formular uma série potencialmente ilimitada de arranjos sociais alternativos (Turner, 2008c: 11-12).

Liminaridad entonces como un momento intermedio; como interface que no connota apenas transición, sino también potencialidad; "it has became clear to us that liminality is not only transition but also potentiality, not only 'going to be' but also 'what may be'" (Turner \& Turner, 1978: 3). Es decir, un dominio en el que no opera la lógica cotidiana signadas por el peso de la estructura social.

De esta manera, el peregrinaje es concebido entonces como suspensión; como espacio refractario; como acontecimiento en potencia.

O processo e estado de liminaridade representam, basicamente, uma negação de muitas, senão de todas, as características da estrutura social pré-liminar e uma afirmação de uma outra ordem das coisas e das relações. A estrutura social não é eliminada, e sim radicalmente simplificada: a ênfase está nos relacionamentos genéricos, e não nos particularistas (Turner, 2008a: 183).

Liminaridad es porque son de estos estados y procesos de los que Turner da cuenta mediante el concepto de communitas, así como de variantes conceptuales como 'estado liminoide' (DaMatta, 2000). Sobre este punto, Carneiro (2004) llama la atención sobre el énfasis de los Turner en el carácter voluntario del acto de peregrinar, lo que haría más apropiada la utilización del término 'liminoide'.

Os Turner (1978) concluem que embora a peregrinação possua alguns atributos de "liminaridade" com os ritos de passagem, a escolha de fazer uma determinada caminhada sagrada é voluntária e, portanto, seria melhor empregar o termo "liminoid". Ou seja, já que a opção pela realização da peregrinação não implica obrigatoriedade, ela não pode ser entendida enquanto "liminal", no sentido atribuído por Van Gennep. Pois o que é enfatizado no primeiro termo é a escolha, o aspecto voluntário da ação e, no segundo, a obrigatoriedade (Carneiro, 2004: 79-80).

Image and Pilgrimage in Christian Culture además de devenir prácticamente la única alternativa al abordaje clásico Durkheimiano en materia de religión marcó una línea de trabajo y modelo casi hegemónico por cerca de veinte años y que al día de hoy continúa siendo de utilidad; "(...) as an institution rather than a place, post-Turnerian pilgrimage came to signify the social modality of 'anti-structure' within anthropological accounts" (Coleman, 2002: 355).

Simon Coleman extrae lo medular de este modelo y su influencia de la siguiente forma:

The considerable influence of the book emerged primarily from its further development and exemplification of a theoretical construct - that of communitas - whose relevance was assumed by the Turners to extend beyond circumscribed social fields into a broader 'Christian culture' that transcended historical, geographical and social boundaries. Communitas described the individual pilgrim's temporary transition away from mundane structures and social interdependence into a looser commonality of feeling with fellow visitors; it clearly drew on metaphors of liminality within rites of passage, but in Image and Pilgrimage also illustrated 'progress from the ludergic liminal to the ergic liminoid' (1978: 36); in other words, was the result of voluntary rather than societally enforced removal from the everyday worldn (Coleman, 2002: 356).

Si bien por un lado el modelo turneriano ofreció (y ofrece) un lugar desde donde pensar el peregrinaje, por otro devino una especie de corsé teórico que no propició la reflexión sobre otras cuestiones o desde otros lugares; las críticas llegaron recién luego de una década. Las más generales señalaban un manejo insuficiente de la movilidad (no obstante ellos la reconozcan) y un énfasis centrado en la cultura del viaje sagrado con foco en la communitas y no tanto en la trayectoria individual del viaje (Coleman \& Eade, 2004; Badone \& Roseman, 2004).

En definitiva, fue el entender al peregrinaje apenas como communitas, como una situación excepcional, lo que motivó la mayor cantidad de reparos. Esa forma de concebir esta práctica espacial, con la atención fijada exclusivamente en la liminaridad, 
terminó por propiciar un considerable descuido de la cotidianeidad social, política y los procesos culturales que también la conforman. Como bien señalan Badone y Roseman, (2004: 4):

many other ethnographic accounts from diverse cultural settings pose a serious challenge to the Turnerian approach by demonstrating that the quality of antistructure and the experience of communitas are largely absent from specific pilgrimage contexts while communitas may be one element of the pilgrimage experience, this social and emotional quality cannot be assumed to exist in all pilgrimages; nor can the concept be used as a master key to unlock the meaning and significance of pilgrimage for all participants in every cross-cultural setting.

Fue precisamente el no perder de vista el carácter sagrado del viaje ${ }^{3}$ y las relaciones que en él tienen lugar, lo que inhabilitó la posibilidad de establecer vinculaciones más estrechas entre peregrinación y turismo o entre peregrinación y prácticas juveniles, o mejor aún, entre peregrinación, consumo, vida cotidiana y prácticas turísticas (Coleman \& Eade, 2004; Badone \& Roseman, 2004; Leite \& Graburn, 2009; Sharpley, 2009). Y a lo que en definitiva las críticas posteriores apuntarán es a trascender la dicotomía sagrado/profando, que de antemano coloca al peregrinaje en relación opuesta e irreductible con otras esferas y prácticas de la vida cotidiana.

Yendo un paso más allá, surgirán autores que discutirán incluso la posibilidad de ampliar el campo semántico de 'sagrado', trascendiendo la órbita de lo estrictamente religioso en lo que refiere al peregrinaje, atendiendo a la construcción de sentidos personales visitando lugares 'profanos', habilitando así una amplia gama connotaciones así como de casos empíricos".

Following Graburn and MacCannell, British sociologists lan Reader and Tony Walter and the contributors to their multidisciplinary volume Pilgrimage in Popular Culture (1993) underscore the parallels between pilgrimage and tourism, arguing that the category "pilgrimage" need not be confined to explicitly religious settings, such as temples, mosques, or cathedrals. These researchers highlight the implicitly religious character of travel to ostensibly secular sites. (...) An important additional series of examples of travel that can be seen to blur distinctions between tourism as "personal quest" and pilgrimage deals with individuals who are engaged in what some term "roots tourism," in reference to Alex Haley's novel Roots (1971) (Badone \& Roseman, 2004: 6-7).

\subsection{Peregrinaciones y Discursos Discrepantes: Contesting the Sacred}

Volviendo a la trayectoria conceptual emprendida en el apartado anterior, la ruptura más formalizada con el modelo de Victor y Edith Turner en lo que se refiere a la institución del peregrinaje en la tradición cristiana fue Contesting the Sacred, compilación Eade y Sallnow publicada en 1991.

El aspecto del modelo clásico que más atención y críticas recibirá será precisamente el carácter excepcional de la práctica del peregrinar y la consiguiente pregunta por nuevas formas de abordar el problema con miras a salir de ese ghetto teorético.

Situados en un contexto teórico y social signado por la fragmentación posmoderna y opuestos a la noción de communitas por entenderla más una idealización que algo emergido de un correlato empírico, los esfuerzos apuntarán a dar cuenta de las peregrinaciones cristianas entendiéndolas como una combinación de personas, textos y lugares, atendiendo a la producción y circulación de discursos discrepantes entre grupos de peregrinos entendidos como 'recipientes vacíos'.

Contesting the Sacred directly opposes the communitas paradigm, focusing instead on the role of major shrines in hosting -and amplifyingdiscrepant discourses among varied groups of pilgrims, this acting as 'empty vessels' that can reflect back visitors' objectified assumptions in sacralized form (Coleman \& Eade, 2004: 4).

La principal idea que subyace además, es la de que el peregrinaje como institución no puede ser hoy día entendido como un fenómeno universal y homogéneo sino que debería ser desconstruido en circunstancias históricas y culturales concretas (Coleman, 2002).

\footnotetext{
3"Edith Turners herself calls pilgrimage 'a kinetic ritual'” (Coleman \& Eade, 2004: 2).

${ }^{4}$ Ver por ejemplo Schramm (2009) que aborda procesos de home-coming de turistas negros americanos en Ghana, o también de Paula y Herédia (2018), que discuten sobre la posibilidad de turistificación de un lugar de memoria como el sitio arqueológico de Cais do Valongo (RJ), susceptible de comportar una dimensión religiosa de matriz africana en su propuesta.
} 
The contestation paradigm reveals its semiotic roots in the argument that varying 'discourses' can be housed under a flexible sacred canopy or 'vessel', through which individuals or groups take account of but do not necessarily specifically interact with each other. It becomes possible to see how the juxtaposition of varied interpretations and practices need not be regarded as, by definition, reflecting over struggles for hegemony in restricted cultural and geographical space (Coleman, 2002: 359).

En tal sentido, las peregrinaciones en general y las que tienen lugar en las sociedades contemporáneas en particular, más que como eventos liminoides, se presentarían como arenas en donde entrarían en conflicto una pluralidad de discursos y sentidos (Carneiro, 2004). De esta manera,

toda peregrinação cria e recria um campo bastante variado de transações, num sistema abrangente de trocas e de permutas dos mais diversos tipos. Ao priorizar mais a dinâmica e multiplicidade do evento, esta nova abordagem revela e destaca todo o poder criativo do ritual, entendido em sua contínua tensão com as ações cotidianas dos grupos sociais envolvidos em sua realização (Carneiro, 2004: 10-11).

Por otro lado, y como ya fue mencionado, la oposición al modelo de communitas llevará a soslayar la carencia del modelo turneriano en no establecer mayores vinculaciones entre peregrinaje, vida cotidiana y prácticas vinculadas al consumo.

Si bien el modelo de Eade y Sallnow significa una alternativa plausible al modelo hegemónico, al tiempo que un avance en lo que refiere a incorporar las críticas formuladas, no por ello estuvo exento de objeciones. En tal sentido, dos de las principales críticas que Coleman y Eade (2004) le formulan a Contesting the Sacred son, por un lado, el hecho de que la tematización del movimiento no está presente, aún permaneciendo aferrados a la clásica equiparación entre 'cultura' y 'lugar'. Por otro lado, llaman la atención sobre el hecho de que la figura del recipiente vacío continua connotando la misma separación que se establece en el modelo clásico; un adentro y un afuera (Coleman \& Eade, 2004).

De esta manera, la presentación de Images and Pilgrimages y Contesting the Sacred en términos de posicionamientos antagónicos, es para Simon Coleman (2002) una simplificación que impide darle a dichas obras una justa dimensión en lo que hace a la utilidad, y en el caso del pensamiento de Turner, a la vigencia. Por otro lado, Coleman sostiene que es esa simplificación la que no permite apreciar con claridad que ambos modelos guardan similitudes entre sí. Así, este autor señala que la idea de ajuste de un lugar sagrado a una multiplicidad de discursos, no es tan distante de la idea turneriana de símbolos dominantes conteniendo una amplia gama de significados semánticamente abiertos (Coleman, 2002).

Y lo más llamativo es que ambos modelos están regidos por metáforas que comportan un 'espacio vacío' a ser llenado con las respectivas visiones sobre lo que sería peregrinaje. En el caso del modelo de los Turner ello desemboca en la idea de communitas y en el caso de Eade y Sallnow en la imagen del lugar sagrado como recipiente a vacío.

La necesidad de dichos espacios teoréticos abiertos obedece a motivos diferentes; en el caso del modelo turneriano, es claramente asociado a la necesidad de marcar una corte entre peregrinación y vida cotidiana, entre sagrado y profano. En el caso de Contesting the Sacred es la idea contraria; la de que el peregrinaje no levanta los conflictos ni los presupuestos mundanos, sino que puede promover contextos para que ellos sean expresados (Coleman, 2002).

\subsection{Coleman y la Antropología en el Peregrinar: una Apertura a la Dimensión Turismológica en los Modelos Posturnerianos}

Si el modelo turneriano devino una suerte de camisa de fuerza teórica que coartó la posibilidad de otros abordajes, Contesting the Sacred consolidó una polarización teorética pasándose a establecer una relación con el trabajo de Turner mediante el énfasis de las diferencias.

Cabería entonces señalarle el mérito a Simon Coleman (2002), en tanto autor y compilador, el de procurar desmontar esa dicotomización y apuntar a reordenar las posiciones. Intentando conciliar el modelo clásico con sus críticas, Coleman apuntará a conjurar la polarización de las posiciones proponiendo un modelo antropológico, no ya del peregrinar sino en el peregrinar.

Ya en una obra anterior junto con el historiador John Elsner, ambos autores se posicionaron frente a Contesting the Sacred, marcando sus confluencias y divergencias al tiempo que Badone y Roseman los vinculan con variados correlatos empíricos. 
For Coleman and Elsner, pilgrimage holds the potential for both communitas and contestation. A similar, flexible approach is exemplified in Jill Dubisch's postmodern and experiential study of the Greek Orthodox shrine of the Church of the Annunciation on the Aegean island of Tinos (1995). Like communitas, liminality from Dubisch's perspective "is not an inherent feature of pilgrimage to Tinos, but is variable, situational and fluctuating" (97). Dubisch's approach is valuable in pointing the way to a nondeterministic use of Turner's categories that would allow the anthropologist to invoke the ideas of liminality and communitas when they illuminate particular ethnographic situations, without the necessity of attempting to fit all pilgrimage-related phenomena into a rigidly preconceived theoretical framework (Badone \& Roseman, 2004: 5).

Sin negar la importancia y necesidad de proporcionar definiciones, el autor y organizador señala que no es del todo productivo reducir el problema a este plano por entender que ninguna definición simple o concreta será lo suficientemente abarcadora. Con ello no se le niega la importancia a la categorización sino más bien se está advirtiendo que difícilmente haya una definición universalizable tanto en el espacio como en el tiempo y ello porque el cúmulo de comportamientos que hace ver personas como peregrinos va a cambiar según los diversos elementos que se tomen en cuenta (Coleman, 2002). De lo anterior se extrae que la situación ideal es que coexistan varios modelos teoréticos y eventualmente que dialoguen. También se extrae un claro posicionamiento crítico a esa tendencia a elaborar categorías a priori e inmanentes para analizar las peregrinaciones (communitas, empty vessels, etc.), apostando más al contexto de cada caso y asumiendo una dimensión performática y evanescente.

A partir de allí, el autor irá abriendo el concepto para transformarlo en un marco que posibilite abordar otras cuestiones tan pertinentes como la propia práctica del peregrinaje (turismo, consumo, migraciones, resortes étnicos, performances) habilitando así la posibilidad de establecer nuevas y variadas relaciones de significado. De esta forma, el peregrinaje no tiene por qué ser una única cosa, sino ante todo una construcción conceptual; una lente para mirar a través:

Sacred travel frequently overlaps with tourism, trade, migration, expressions of nationalism, (...)
The point is that we must not adopt the rather western habit of treating the category of religion, and everything associated with it, as ideally an autonomous, isolated realm of human activity, and therefore as an autonomous, isolated realm of anthropological theorizing (Coleman, 2002: 363).

Este contexto habilita un movimiento epistémico tan audaz como pertinente y necesario que consiste en pasar de estudiar las peregrinaciones y sus personas, para estudiar a las personas como peregrinos y todo ello sin negarle el valor a los modelos precedentes; por otro lado, este movimiento favorece una redimensión del objeto;

(...) the most valuable work in this area is that which looks outward, making points about human behavior through using 'pilgrimages' as a case-study rather than focusing on the institution itself as a firmly bounded category of action (Coleman, 2002: 363).

También la antropología de la religión en tanto subdisciplina saldrá fortalecida en este movimiento al ser ampliados su dominio y alcance. Es posible pensar que en este cambio de perspectiva, también la noción de religión y su vinculación con lo sagrado se haya visto modificada, quedando cada vez menos restringida a una esfera autónoma y especializada (Hervieu-Léger, 2005).

Tomando en cuenta lo anterior, deviene perfectamente lícito por ejemplo atender a las formas de corporización de la creencia (embodiment) en el contexto de la peregrinación, o a las vinculaciones entre memoria, desplazamiento e identidad religiosa, cuando no ya las diversas formas de pertenencia inclusive en el contexto del turismo religioso. O como en el libro organizado por Badone y Roseman (2004), o por el de Coleman y Eade (2005), que en gran medida se proponen atender a las diversas formas de movimiento (corporificado, metafórico, imaginado) como todas ellas constitutivas de muchas posibles formas de peregrinajes, al tiempo que prestar atención a cómo se imbrican las peregrinaciones con otras esferas de la vida cotidiana como el consumo y principalmente el turismo y la recreación.

The recent literature on pilgrimage has shown that the framing of pilgrimage within the discourse and practice of the tourism industry is far from unusual. Rigid distinctions between (serious) pilgrims-always on a journey to a sacred site-and (playful) tourists-always on a trip to places of secular pleasure-have become 
blurred. Centres of religious pilgrimage such as Lourdes in France have virtually grown into tourist attractions (Scharmm, 2004: 136)

Consecuencia directa de este movimiento ha sido el acercamiento en las últimas décadas entre la antropología y los estudios del turismo religioso, no solamente en el mundo anglosajón o francoparlante sino también en Iberoamérica (Germiniani, 2006; Germiniani, 2009; Jácome, 2016; Mora Torres et al., 2017). En ese sentido, se buscarán modelos de análisis integradores para la práctica del peregrinar, poniendo en diálogo ambas tradiciones. Así, en la misma dirección que los autores de Contesting the Sacred y los estudios de Steil, enfocados principalmente en el caso brasilero y en el tradicional Camino de Santiago, Carneiro (2004; 2012) asume que en términos analíticos las prácticas de peregrinaje y las prácticas turísticas se presentan como

duas estruturas de valores e sentidos distintas. No entanto, no nível empírico, estes campos aparecem sempre imbricados, tornando suas fronteiras bastante fluidas e híbridas, constituindo-se em estruturas de significados que se articulam e se combinam de várias maneiras formando arranjos sempre renovados e em permanente mutação. De acordo com Steil (1999), nesta imbricação podemos ver surgir um novo campo que podemos denominar de turismo religioso (Carneiro, 2004: 92).

A pesar de que la antropología haya demorado casi cuarenta años para percibir primero e integrar después una dimensión turismológica en sus modelos sobre el peregrinar ${ }^{5}$, esa incorporación significó una importante contribución a los estudios sobre el turismo religioso; prueba de lo anterior pueden ser los trabajos de Germiniani o Jácome donde las autoras realizan una aproximación a las interfaces entre turismo y peregrinación moderna desde su mirada disciplinar situada en los estudios turísticos (Germiniani, 2006; Germiniani, 2009; Jácome, 2016). En ese sentido, es interesante ver la autopercepción que se tiene desde el turismo religioso y el reconocimiento de los aportes de las ciencias humanas al tiempo que de la imbricación con las prácticas religiosas.

O turismo religioso tem vindo a consolidar-se nas últimas décadas quer pelo uso generalizado da terminologia (Silveira, 2004, 2007) como subtipo do turismo, quer pelo aumento do número de turistas e crescente impacto na atividade econômica local, quer ainda pelo reconhecimento acadêmico (Richards e Fernandes, 2007) e crescente interesse dos investigadores de diferentes áreas do saber, da antropologia à sociologia, da geografia à economia (Dias, 2010; Guillaumon, 2012). Apesar disso, não há consenso sobre o próprio conceito de turismo religioso, sendo que na prática há uma relação quase umbilical entre turismo e religião (Prazeres \& Carvalho, 2015: 1145-46).

Ya para el caso concreto de las peregrinaciones,

Considerando al turismo y a la peregrinación como movilidades humanas con significados diferentes, el turismo religioso es un concepto utilizado frecuentemente en las publicaciones sobre el tema para referirse a viajes religiosos que presentan algunas características del turismo convencional, o a desplazamientos hacia lugares considerados como sagrados independientemente de la motivación de los visitantes. (...) Ya que el turismo religioso refleja teóricamente las múltiples y cambiantes motivaciones del viajero, cuyos intereses y actividades pueden moverse del turista al peregrino y viceversa, incluso sin que el individuo se dé cuenta del cambio (Smith, 1992), frecuentemente es utilizado en la literatura turística como un concepto que engloba las relaciones entre los dos tipos de desplazamiento. Por tanto, "turismo religioso" es un término relevante para la búsqueda de publicaciones que discuten las convergencias y divergencias del turismo con la peregrinación (Mora Torres et al., 2017: 89-90).

Tal como establece Gustavo Ribeiro (2011: 17), "um dos desafios nas ciências sociais é encontrar os cenários etnográficos adequados para dialogar com diferentes discussões teóricas e fazê-las avançar." De esta manera y a los efectos de este texto, queda de manifiesto en los autores y modelos posturnerianos la intención y la necesidad de llamar la atención para los vínculos explícitos entre turismo y peregrinaciones, incluyendo así una dimensión turismológica a sus abordajes.

\footnotetext{
${ }^{5}$ Si bien ya sobren finales de los ' 70 en autores como MacCannell o Cohen, comienzan a verse los primeros movimientos tendientes a vincular de alguna forma las prácticas turísticas con las peregrinaciones (Carneiro, 2004).
} 
Si se ha optado por trazar esta trayectoria conceptual mostrando detenidamente el contexto que habilitó la apertura de la noción de peregrinaje, posibilitando la inclusión de una dimensión turismológica, es porque es este tipo de modelo posturneriano el que rige los análisis hasta el día de hoy.

\section{CORRELATOS EMPÍRICOS}

Quisiera presentar a continuación algunos correlatos empíricos de la discusión desarrollada más arriba. El criterio de selección fue la forma en que son estudiadas las peregrinaciones desde la antropología, sea vinculándolas a las prácticas turísticas, sea trascendiendo la órbita de lo sagrado, sea incluyendo una dimensión turismológica. También por el hecho de que algunos de estos trabajos fueron emblemáticos de este cambio de perspectiva y ejercieron una influencia importante en las producciones y abordajes posteriores. Concretamente me centraré en el caso brasilero trayendo a colación los trabajos de Steil (2003) y Carneiro (2004) abordando diversas formas de peregrinaciones, así como un estudio de Mitchell (2001) sobre la visita de mormones británicos a lugares sagrados en el estado de Utah.

\subsection{Mormones y Romeros: algo más que peregrinos}

Hildi Mitchell en su texto 6 aborda la peregrinación de los Mormones británicos a Salt Lake City atendiendo a las formas de corporificación del conocimiento y a la dimensión material del conocimiento religioso.

Este es el contexto de una peregrinación en donde lo histórico, y aunque la autora no lo explicite, también lo turístico están atravesados por lo sagrado; cacaso los mormones peregrinos no construyen una 'mirada turística'? (Urry, 1996).

La historia vivida por los peregrinos es historia vivida como teología y en ella priman experiencias físicas, sensoriales y afectivas que tienen más que ver con una verdad religiosa que con una verdad historiográfica;

visiting Mormon historical sites, museums and key buildings is one way in which Mormons are able to participate actively in their theology and cosmology, and this follows the same pattern as Mormon engagement with other material objects. Mormon engagement with the material remnants and reminders of their history through embodied memories of their engagement with the objects, buildings and narratives of their theology (Mitchell, 2001: 9).

De esta manera tiene lugar el encuentro con una diversidad de objetos, lugares y textos que cada peregrino ha ido interiorizando y construyendo no bajo la forma de presencia, sino de represencia (Said, 2002); un lugar que en lo previo es "menos un lugar que un topos, un conjunto de referencias, un cúmulo de características" (Said, 2002: 243) originado en construcciones personales estrechamente vinculadas con lo vivencial. $Y$ es precisamente en ese encuentro entre el contenido vivencial que porta el peregrino y un espacio espectacularizado (Falero, 2014) que acontece una materialización y una personificación de la verdad religiosa.

Es más que claro que para dar cuenta de las formas de conocimiento presentes en esta experiencia es preciso trascender aquellos modelos que se centran en lo meramente intelectual para pasar a aquellos que conciben que el conocimiento se da con el cuerpo en su totalidad como por ejemplo Thomas Csordas (1990) y su noción de Embodiment. Es decir, no tanto como mera representación sino como vivencia y corporalidad.

Otro ejemplo ya más cercano a nosotros es el trabajo de Carlos Steil en el santuario del Bom Jesus da Lapa en el Estado de Bahia, Brasil. Allí se asume que "turismo" y "peregrinación" condensan dos estructuras de significados que coexisten y permanentemente son actualizadas en el evento. Así en la romería, más que un antagonismo, se da una tensión entre dos formas de sociabilidad (Steil, 2003).

Junto a muchos romeros que viven la peregrinación bajo el signo de la communitas, llegando en camiones abarrotados de personas, estableciendo lazos de solidaridad entre desconocidos, pasando sin demasiado confort durante el trayecto y también durante el evento, opera también principalmente entre los pobladores del lugar y el clero, un cúmulo de ideales regulativos que establecen que la romería debería estar pautada por el orden, la higiene y el confort, cuando no ya directamente por normas más próximas a una lógica turística; $y$ de hecho hay también una porción de romeros-turistas, como los llama el

\footnotetext{
${ }^{6}$ Si bien su versión original es del 2001, fue incluido en la compilación de Coleman y Eade (2005).
} 
autor, que viaja cómodamente, se instalan en hoteles y llega al lugar buscando otras cosas (Steil, 2003). De ello resulta que las categorías de "turista" y "romero" pueden llegar a ser bastante inestables llegando incluso a confundirse la una en la otra;

observarmos que existe uma miscelânea de atos religiosos e turísticos praticados pela mesma pessoa, de modo que se torna muito difícil saber se estamos diante de um turista ou de um romeiro (Steil, 2003: 251).

El autor retomando precisamente la metáfora de los autores de Contesting The Sacred, quienes conciben a la peregrinación como una especie de recipiente vacío capaz de contener una diversidad de prácticas y sentidos, entiende que esa condición la que le permite que ambas lógicas se articulen mediante la oposición, aunque sin por ello excluirse (Steil, 2003).

\subsection{Nuevas Rutas de Peregrinación en Brasil: turismo como mediación de lo sagrado}

Sandra de Sá Carneiro (2004), influenciada por Eade y Sallnow y las investigaciones de Steil sobre el Camino de Santiago de Compostela, analiza el caso brasilero de nuevas rutas emergentes y lo hace desde su inicio, a comienzos del año 2000. Estas rutas son inspiradas precisamente en el modelo del Camino de Santiago e incluso sirven como una forma de instancia previa para una posterior visita y realización del propio itinerario de Compostela.

Rutas inventadas aunque no por ello 'inauténticas'; tan inventadas y reinventadas como el propio Camino de Santiago. Al respecto, el propio Steil señala que a partir de la última década del Siglo XX la peregrinación hacia Santiago de Compostela ha experimentado una importante revitalización

[...] entre um número crescente de indivíduos de classes médias na Europa e nas Américas. Vividas como uma performance crítica ao racionalismo e ao consumismo da modernidade capitalista, estas novas peregrinações geralmente vêm associadas a valores ecológicos e alternativos ao sistema dominante. Esta reinvenção dos Caminhos de Santiago de Compostela, por sua vez, enseja a criação de outras rotas de peregrinação fora da Espanha que se espelham no modelo de Santiago na Espanha (Steil, 2018: 12)
En el caso brasilero, esas nuevas rutas identificadas por Carneiro, que las entiende como un correlato de esa revitalización, son: el Caminho do Sol en São Paulo, el Caminho da Luz en Minas Gerais, el Caminho das Missões en Rio Grande do Sul, los Passos de Anchieta en Espírito Santo y el Caminho da Fé en São Paulo.

Como fue mencionado en la sección anterior, esta autora, asumiendo a las peregrinaciones y al turismo como dos estructuras de significado que habilitan distintas formas de vivenciar esas prácticas así como también de transformarlas, y asumiendo también el presupuesto de Steil en relación a que hay una dimensión performática en dos planos que se fusionan, el del actor y el del espectador, y asumiendo además que el plano de las prácticas turísticas apunta para el espectáculo y en definitiva hacia la exterioridad, la autora entiende que la principal diferencia entre turismo y peregrinación pasa por una cuestión de mayor o menor externalidad en la mirada y en el grado de inmersión que estas experiencias posibilitan. De allí que a pesar de haber elementos en común, los peregrinos elaboran autodenominaciones excluyentes, poniendo de manifiesto el elemento transformador de su experiencia (Carneiro, 2004).

En ese sentido la autora se dedicará a profundizar sobre esa articulación y sus consecuencias, asumiendo ambas estructuras en interface. Entre las características comunes que engloban estas rutas, están la posibilidad de recorrer un camino de paisajes bucólicos, pernoctar en antiguas estancias, visitar lugares con valor patrimonial, conocer ámbitos con valor memorial e histórico, rehacer el itinerario que en otro tiempo fue realizado por un personaje que es histórico y mítico al mismo tiempo, conocer lugares con el afán de revivir un pasado entre mítico e histórico. Todo ello en un ambiente propiciado para la introspección y sin descuidar el confort, la seguridad y los detalles propios de un producto turístico. La propia autora siguiendo a Steil reconoce que las cinco rutas se enmarcan dentro de lo que este éste denomina turismo religioso.

Estamos diante de experiências rituais que no sentido "mais tradicional" poderiam ser denominadas de peregrinações, mas que no contexto atual de uma sociedade moderna (brasileira), se constituem em polos de atração de pessoas, justamente por assumirem também, em sua expressão, um aspecto 
turístico e de lazer. Na construção dessas espacialidades, a relação entre religião e turismo pode ser considerada como um dos elementos centrais (Carneiro, 2004: 93).

Quienes emprenden esos caminos lo hacen movidos en gran medida por un afán de 'autotransformación'; esta experiencia que es presentada como profunda, personal e intransferible, es también experiencia desinstitucionalizada en lo religioso, al tiempo que institucionalizada en lo turístico. Así, al participar de ambas estructuras en interface, peregrinación y turismo, a estos visitantes en tránsito la autora propone entenderlos como 'peregrinos-turistas'.

Desta forma, emerge uma nova categoria de peregrinos-turistas ou de turistas religiosos, que se diferenciam dos peregrinos tradicionais não apenas pelo conjunto de motivações que os levam a realizar a peregrinação, mas, sobretudo, pelas estruturas de significados nas quais estão inscritas suas experiências (Carneiro, 2004: 93)

A los efectos de los objetivos del presente texto cabrían dos últimos aspectos a resaltar que se desprenden de lo anterior. En primer lugar, la autora demuestra que estos cambios en las dinámicas de las peregrinaciones son correlatos de las profundas transformaciones que han tenido lugar en el campo religioso; en ese sentido, la principal característica de estas nuevas configuraciones del peregrinar, es la emergencia del turismo (y de las actividades vinculadas al ocio y al consumo en general), como mediador de lo sagrado.

Por otro lado, que el modelo religioso operante es más propio de las religiones del self, signadas por una espiritualidad difusa y desinsitucionalizada, propias de esta modernidad tardía, que de un modelo tradicional anclado en dinámicas grupales y eso lo haría alejar de la lógica de la communitas.

Trata-se assim de considerar a essencial heterogeneidade do processo de peregrinação suprimido ou negado por outras perspectivas analíticas. Portanto, adoto como perspectiva analítica aquela que entende a peregrinação como lugar de encontro e muitas vezes de competição de discursos religiosos e seculares (EADE e SALLNOW, 1991). O enfoque multidimensional proposto por estes autores implica uma mudança na compreensão do sagrado e da peregrinação. Realizar esses caminhos não é uma experiência que tem um significado unívoco, autônomo e plenamente constituído, e sim que se constitui como projeção, convergência e elaboração a partir de diferentes universos de significado (Carneiro, 2012: 70).

\section{PALABRAS FINALES: DE LA ANTROPOLOGÍA DEL PEREGRINARALAANTROPOLOGÍA EN EL PEREGRINAR}

Por tratarse de un texto antropológico publicado en un ámbito turismológico, y dirigido principalmente a un público enmarcado en los estudios turísticos, su cometido fue teórico y didáctico al mismo tiempo. Por un lado pretendí mostrar de manera sistematizada los principales derroteros que han organizado y organizan hasta el presente la mirada antropológica en torno al fenómeno de las peregrinaciones. Y por otro, dejar en evidencia el momento y el contexto de la producción de conocimiento en ese campo de saber, en el que una dimensión turismológica comienza a ser considerada y desarrollada en estos modelos. A partir de aquí, se abrirá un fecundo ámbito de nuevas posibilidades conceptuales y abordajes diversos que dura hasta el presente.

Así, a través del itinerario teórico trazado y de los correlatos empíricos presentados, han quedado en evidencia dos aspectos. En primer lugar, el punto de inflexión en el que los abordajes antropológicos de las peregrinaciones comienzan a redimensionar su objeto y pasan a incorporar una dimensión turismológica en sus modelos de análisis, complejizándolos y propiciando un diálogo más estrecho y fluido con los estudios turísticos. Luego, y consecuencia de lo anterior, la emergencia de nuevos desafíos teóricos y metodológicos que surgen al pretender realizar un abordaje más cabal de este tipo de prácticas; al comenzar a ser tenidas en cuenta las variadas dimensiones que la práctica del peregrinaje comporta, el consumo, el turismo y los diversos usos del tiempo libre no podrían permanecer ajenos. Yendo un paso más allá, para el caso de antropólogos como Carneiro o Steil, este ámbito propicia el surgimiento del 'turismo religioso' en tanto lugar de estructuras en diálogo (no exentos de tensiones) y en el cual pueden pensarse los ejemplos citados. 
Em termos analíticos, a peregrinação e o turismo se apresentam como duas estruturas de valores e sentidos distintas. No entanto, no nível empírico, estes campos aparecem sempre imbricados, tornando suas fronteiras bastante fluidas e híbridas, constituindo-se em estruturas de significados que se articulam e se combinam de várias maneiras formando arranjos sempre renovados e em permanente mutação (Carneiro, 2004: 92).

Entiendo que esta manera de recontextualizar el objeto posibilita con más facilidad entender al turismo como algo más que una serie de prácticas vinculadas apenas con la mercantilización del tiempo libre, pasando a ser entendida como una práctica espacial signada por el movimiento voluntario situándose así junto con otras prácticas de la movilidad (Coles et al., 2005); la ventaja que presenta este esquema es habilitar de manera más fluida las eventuales interfaces entre turismo y otras modalidades de desplazamiento (Carneiro, 2004; Coles et al., 2005; Barretto, 2009).

A lo anterior habría que agregarle un contexto de modernidad religiosa caracterizado por una crisis institucional de las religiones tradicionales tanto en lo que hace a la regulación de la creencia así como en la transmisión de la misma; es decir, una sociedad secularizada en que la desregulación de la creencia se masifica y comienzan a emerger nuevas formas autónomas de individualización y de subjetivación de las creencias y de las prácticas, alejadas de la órbita del control institucional (Hervieu-Léger, 2005). Así, hoy más que nunca, el peregrino realiza su viaje de la manera que mejor lo entienda sin estar demasiado pendiente de prescripciones institucionales.

Ya en relación a intereses disciplinares específicos, es sobre este marco de fondo que operan las preguntas que señalan fronteras: dónde comienza y dónde termina la órbita de la antropología de la religión y la de los estudios turísticos; qué elementos atender y cómo relacionarlos, y más aún, cómo están trazadas en este contexto esas prácticas materiales y discursivas, históricas por antonomasia, que configuran 'lo religioso' y/o 'lo sagrado'. Parafraseando a Ervin Goffam cabría preguntarse sobre qué es lo que en la actualidad pretende verse, si los peregrinajes y sus personas o las personas y sus peregrinajes.
Mora Torres et al. (2017) han dejado de manifiesto que la antropología ha sido una de las ciencias humanas que más han reflexionado y aportado al estudio de las peregrinaciones en general, y a su vínculo entre éstas y el turismo en particular; ello en gran medida ha quedado de manifiesto en las páginas anteriores.

\section{REFERENCIAS}

Badone, E. \& Roseman, S. (2004). Approaches to the Anthropology of Pilgrimage and Tourism. En: Badone, E. \& Roseman, S. Intersecting Journeys. The Anthropology of Pilgrimage and Tourism. (pp. 1-23). University of Illinois Press. USA.

Barretto, M. (2009). Interfaces entre Turismo e Migrações: uma Abordagem Epistemológica. Passos. Revista de Turimo e Patrimônio Cultural. 7(1), 1-11.

Bauman, Z. (1999). La Globalización. Consecuencias Humanas. FCE. Buenos Aires.

Carneiro, S. de S. (outubro de 2004). Novas Peregrinações Brasileiras e suas Interfaces com o Turismo. Ciencias Sociales y Religión/Ciências Sociais e Religião, Porto Alegre, 6(6), 71-100.

Carneiro, S. de S. (Jan./Jun de 2012). As Peregrinações como Atrações Turísticas. Espaço e Cultura. UERJ, RJ, (31), 66-79.

Clifford, J. (1999a). Prólogo: in media res. En: Clifford, J. Itinerarios transculturales. (pp. 11-25). Gedisa editorial. España.

Clifford, J. (1999b). Prácticas espaciales: el trabajo de campo, el viaje y la disciplina de la antropología. En: Clifford, J. Itinerarios transculturales. (pp. 71-119). Gedisa editorial. España.

Coleman, S. (2002). Do you believe in pilgrimage?: Communitas, contestation and beyond Anthropological Theory. 2(3), 355-368.

Coleman, S. \& Eade, J. (2004). Reframing Pilgrimage. Routledge. USA.

Coles, T.; Duval, D.; \& Hall, M. (2005). Sobre el Turismo y la Movilidad en Tiempos de Movimiento y Conjetura Posdisciplinar. Política y Sociedad. 42(1), 85-99.

Csordas, T. J. (Mar. 1990). Embodiment as a Paradigm for Anthropology. Ethos, 18(1), 5-47.

DaMatta, R. (2000). Individualidade e liminaridade: considerações sobre os ritos de passagem e a modernidade. Mana [online], 6(1), 7-29.

Durkheim, É. (2008 [1912]). Las formas elementares de la vida religiosa. Paulus. São Paulo.

Paula, Á. T. de; Herédia, V. (Jan./Abr. 2018). A “Turistificação" de um lugar de Memória É Possível? Um Estudo Sobre o Sítio Arqueológico do Cais do Valongo (Rio De Janeiro, Brasil). Revista Anais Brasileiros de Estudos Turísticos/ ABET, Juiz de Fora, 8(1), 8-22.

Eade, J.; Sallnow, M. (1991). Contesting the Sacred. The Anthropology of Christian Pilgrimage. Routledge. London/New York. 
Falero, A. (2014). Producción del espacio urbano y captación del turismo en un contexto de transformaciones sistémicas globales, trabajo contenido. En: Falero, A.; Campodónico, R. (comp.) El Turismo Bajo la Lupa Académica. (pp. 89 - 105). Biblioteca Plural, CSIC. Montevideo.

Germiniani, H. C. (2006). A "Santiago de Compostela" Brasileira: Religião, Turismo e Consumo na Peregrinação pelo Caminho da Fé. Tese apresentada ao Programa de PósGraduação em Ciência da Religião. UFJF-MG. Brasil.

Germiniani, H. C. (1 Semestre de 2009). Turismo-Religioso. No Caminho da fé. Revista Eletrônica de Turismo Cultural. 03(01), 75-94.

Hannerz, U. (Apr. 1997). Fluxos, fronteiras, híbridos: palavraschave da antropologia transnacional. Mana. 3(1). Rio de Janeiro.

Hervieu-Léger, D. (2005 [1999]). O peregrino e o convertido. A religião em movimento. Gradiva. Lisboa.

Jácome, G. A. (Set./Dez. 2016). Turismo Religioso: O Caso do Jubileu de Conceição do Mato Dentro e do Impacto da Mineração na Região (Minas Gerais/Brasil). Revista Anais Brasileiros de Estudos Turísticos/ ABET, Juiz de Fora, 6(3), 30-43.

Jamal, T.; Robinson, M. (2009). Introduction: The Evolution and Contemporary Positioning of Tourism as a Focus of Study." In: The SAGE Handbook of Tourism Studies. (pp. 1-16). SAGE Publications. LTD. London.

Leite, N.; Graburn, N. (2009). Anthropological Interventions in Tourism Studies. In: The SAGE Handbook of Tourism Studies. (pp. 35-64). SAGE Publications. LTD. London.

Lash, S.; Urry, J. (1998 [1994]). Economía de Signos y Espacio. Sobre el Capitalismo de la Posorganización. Amorrortu Editores. Bs. As.

Mitchell, H. (April. 2001). 'Being there'. British Mormons and the History Trail. Anthropology Today, 17(2), 9-14.

Mora Torres, V. M.; Serrano Barquín, R. Del C.; \& Osorio García, M. (2017). El vínculo turismo-peregrinación. Estudios y Perspectivas en Turismo, 26, 86-106.

Ribeiro, G. L. (2011). Antropologia da Globalização. Circulação de Pessoas, Mercadorias e Informações. Série Antropologia - Departamento de Antropologia UNB,N $N^{\circ} 435 . \quad B r a s i l i a . \quad C o n s u l t a d o$ en http://vsites.unb.br/ics/dan/Serie435empdf.pdf
Ribeiro, M. (Jul./Dez. 2017). Turismo em Tempos de Modernidade Líquida. Revista Latino-Americana de Turismologia / RELAT, Juiz de Fora, 3(2), 8-23.

Prazeres, J.; Carvalho, A. (Octubre de 2015). Turismo Religioso: Fátima no Contexto dos Santuários Marianos Europeus. En: Pasos. Revista de Turismo y Patrimonio Cultural. 13(5), 1145-1170.

Salazar, N. (2018). Theorizing mobility through concepts and figures. Tempo Social. Revista de Sociologia da USP, 30(2), 153-168.

Said, E. W. (2002[1978]). Orientalismo Debate. España.

Schramm, K. (2004). Coming home to the Motherland: pilgrimage tourism in Ghana. In: Coleman, Simon; \& Eade, John. Reframing Pilgrimage. (pp. 135-152). Cultures in motion. Routledge. London. New York.

Sharpley, R. (2009). Tourism, Religion, and Spirituality. In: The SAGE Handbook of Tourism Studies. (pp. 237-253). SAGE Publications. LTD. London.

Steil, C. (2003). Romeiros e turistas no santuário de Bom Jesus da Lapa. Horizontes Antropológicos. Porto Alegre, 9(20).

Steil, C. A. (jan./abr. 2018). Editorial: Peregrinação: Sentidos e Práticas. Horizonte. PUC - Belo Horizonte, 16(49), 10-13.

Turner, V. (1974 [1969]). O Processo Ritual. Estrutura e Antiestrutura. Editora Vozes. Petrópolis - RJ.

Turner, V. (2008a [1971]). Peregrinações como procesos sociais. En: Turner, V. Dramas, Campos e Metáforas. Ação Simbólica na Sociedade Humana. (pp. 155-214). Editora da Universidade Federal Fluminense. Niterói, R.J.

Turner, V. (2008b [1974]). Metáforas da antiestrutura na cultura religiosa. En: Turner, V. Dramas, Campos e Metáforas. Ação Simbólica na Sociedade Humana. (pp. 253-277). Editora da Universidade Federal Fluminense. Niterói, RJ.

Turner, V. (2008c [1974]). Apresentação. En: Turner, V. Dramas, Campos e Metáforas. Ação Simbólica na Sociedade Humana. (pp. 11-17). Editora da Universidade Federal Fluminense. Niterói, R.J.

Turner, V.; \& Turner. E. (1978). Image and Pilgrimage in Christian Culture. Antropological Perspectives. Basil Blackwell. Oxford. UK.

Urry, J. (1996 [1990]). O Olhar do Turista. Lazer e Viagens nas Sociedades Contemporâneas. SESC - Studio Nobel. São Paulo. 